\title{
Poliglobulia neonatal. Revisión de 110 casos
}

\author{
Dr. Walter Rivera M. ${ }^{1}$; Dr. Ignacio Sánchez D. ${ }^{2}$ : Dr. Felipe Cavagnaro S.M. ${ }^{2}$; \\ Dr. Augusto Winter G. ${ }^{1}$; Dr. José Luis Tapia I. ${ }^{1}$; Dta. Gabriela Juez G. ${ }^{1}$ \\ Neonatal polycythemin: 110 cases reviewed
}

\begin{abstract}
Between January 1984 and June 1986 I 10 cases of neonatal polycythemia were detected. Fhere were $35.4 \%$ small for dates and $12.7 \%$ large for dates cases in the study population. Matemal toxemia and gestational diabetes were the most frequently associated maternal diseases with $17.2 \%$ and $6.2 \%$ respectively. Down syndrome was found on six babies (5.2\%). An early diagnosis of polycythemia (0-2 hours) was made in $52.8 \%$ of the cases: $60.2 \%$ werc symptomatic, with plethora $(78.4 \%)$, cyanosis, jitteriness, hypotonis and tachypnea were less frequently encountered. In $45(40.9 \%)$ cases partial exchange was indicated; $11(24.4 \%)$ were done through the umbilical vein and 34 $(75.6 \%)$ through peripheral veins. Other associated diseases were hyperbilirrubinemia in 40 cases $(36.3 \%)$, hypoglicemia $7(6.4 \%)$ and sepsis $6(5.5 \%)$. There was only one death attributed to multiple anomalies. These results are simjlar to other series published, suggesting that peripheral veins can be used as an alternative for partial exchange.

(Key words: neonatal polycythemia, venous hematocrit, partial exchange.)
\end{abstract}

La presencia de poliglobulia en el período neonatal ha sido bien documentada. Se define por la existencia de hematocrito venosos igual o mayor a $65 \%$ en la primera semana de vida, pudiendo o no acompañarse de síntomas. Se le reconoce como una adoptación fisiológica al avance de la edad gestacional del feto ${ }^{1,4}$. Diversos factores condicionan esta afección: prenatales, que tienen como hecho común la hipoxia; intra parto, también como respuesta a menor oxigenación y post-natales, transfusión placento-fetal ${ }^{\mathbf{L}}{ }^{3}$. La poliglobulia se asocia a una serie de enfermedades importantes del periodo neonata] como hiperbilirrubinemia, hipoglicemia, hipocalcemia. alteraciones de la coagulación, enterocolitis necrotizante $e^{5,6}$.

1. Departamento de Pediatría, Escuela de Medicina. Pontificia Universidad Católica de Chile.

2. Becados Departamento Pediatría, Escuela de Modicina. Pontificia Universidad Católica de Chile.
Varias publicaciones recientes muestran avances en el conocimiento de la patogenia y manejo de la poliglobulia; sin embargo, aún permanecen varios tópicos en discusión, cómo por ejemplo la viscosidad del plasma y la via para realizar la eritroféresis $^{3,7,8}$. En la literatura nacional de los últimos años no hay referencias a este tema. El propósito de este estudio es describir las características clínicas, diagnóstico y manejo de la poliglobulia neonatal observadas en una unidad de neonatología durante 30 meses.

\section{MATERIAL Y METODO}

Se revisaron las fichas clínicas de los 5.820 recién nacidos (RN) entre el $1^{\circ}$ de enero de 1984 y el 30 de junio de 1986 en la maternidad del Hospital Clinico de ta Pontificia Universidad Católica de Chile. Se pesquisaron 116 casos de poliglobulia neonatal $(1,99 \%)$, de los que se analizaton 110 por haber 6 fichas incomple. tas. 
Se tomaron muestras de sangre de venas del dorso do la mano, en todos los recién nacidos con síntomas sospechosos (plétora, temblor, cianosis, hipotonía, convulsiones) y a todos los considerados con riesgo de poliglobulia: (pequeños para la edad gestacional [PFG], grandes para la edad gestaclonal [GEG], hijos de madre diabética, síndrome de Down, ligadura tardía del cordón umbilical), calificándolos como poliglobulia si el hematocrito era igual o mayor que $65 \%$, como asinismo a cualquier RN en cuyas muestras de sangre, tomadas por otros motivos, el hematocrito capilar fuese mayor de $70 \%$. En la mayoría de los casos la muestra de singre se tomó antes de las dos horas de vida, se centrifugó a $3.600 \mathrm{rpm}$ durante tres minutos en microcentrífuga International Modelo M.B. (International Equipment Co. Boston Mass., USA).

Los RN fueron clasificados según edad gestacional (EG), peso, talla y circunferencia craneana, en AEG (adecuados para la edad gestacional), $\mathrm{PEG}, \mathrm{GEG}^{9}$, registrando de la ficha materna edad, paridad y enfermedades del embarazo. De la observación neonatal se obtuvo información sobre malformaciones congénitas, anomalías cromosómicas, manifestaciones clínicas, tipo de tratamiento recibido, vía usada para eritroféresis, complicaciones, días de hospitalización y morbilizad concomitante.

En los casos en que se ropuirió exitroféresis, ésta se rcalizó por vía venosa central (vena umbilical) o periférica (basílica, cefálica o mediana). En las realizadas por vía central se utilizó la técnica habitual de exanguíneo-transfusión. Cuando se utilizó via periférica, la técnica consistió en extraer sangre a través de la vena y al mismo tiempo inyectar plasma, con bomba de infusión continua, por una vena distinta a la de extracción. La cantidad de sangre para extraer se calculó por la fórmula de $\mathrm{Oski}{ }^{1}$.

En el análisis de la morbilidad conounitante se usaron las siguientes definiciones: hipoglicemia: glicemia $\leqslant 40 \mathrm{mg} / \mathrm{dL}$; hipocalcemia: calcio $\leqslant 7 \mathrm{mg} / \mathrm{dL}$; trombocitopenia: $<150.000$ plaquetas $/ \mathrm{mm}^{3}$; hiperbilirrubinemia: bilirrubina sérica mayor de $5 \mathrm{mg} / \mathrm{dL}$ en primeras 24 horas, mayor de $10 \mathrm{mg} / \mathrm{dL}$ entre 24 y 48 horas $y$ mayor de $15 \mathrm{mg} / \mathrm{dL}$ en cualquier momento

\section{RESULTADOS}

De los 110 casos analizados $53(48,2 \%)$ eran hombres; $28(255 \%)$ tenian menos de 38 semanas de EG destacando un paciente de $34 \mathrm{y}$ tres de 35 semanas; $80(72,7 \%)$ eran de término, y 2 (1,8\%) de post-término $>42$ semanas). En la tabla 1 se comparan las edades gestacionales en la población estudiada con la del total de RN vivos del periodo: 39 casos $(35,4 \%)$ eran PEG; 14 GEG $(12,7 \%)$ y 57 AEG $(51,8 \%$. La tabla 2 compara las distribuciones según el peso para la EG en los pacientes $y$ el total de nacidos vivos del período. La edad materna fue en promedio 28,8 años (rango 17 a 44), la paridad promedio de
2,06 hijos ( 1 a 6 ). La via de parto fue vaginal en 93 casos $(97,8 \%)$ y cesárea en $22(20,2 \%)$, en las pacientes, mientras en el total de los nacidos vi. vos fue de $73,9 \%$ y $26,1 \%$, respectivamente. Dentro de las enfermedades maternas, el sindrome hipertensivo del embarazo (SHE) ocurrió en 20 casos (17,2\%) y diabetes gestacional en 7 casos (6\%).

Seis afectados con poliglobulia tenian síndrome de Down $(5,2 \%)$; en cambio, entre el total de $\mathrm{RN}$ vivos la incidencia del sindrome fue de $12\left(0,2 q_{0}\right)$.

El diagnóstico fue precoz antes de las 2 horas en 57 casos $(52,8 \%)$, el promedio fue de 8,67 horas $(1,5-108)$. La forma de presentación clínica fue asintomática en 43 casos $(39,8 \%)$ y sintomática en $67(60,2 \%)$, destacando rubicun. dez en $51(78,4 \%)$ y con menor frecuencia cianosis, polipnea, temblor, hipotonía (tabla 3).

El valor del hematocrito (Hto) fue de 65 a $69 \%$ en 60 casos (55\%), y de $70 \%$ o más en 50 (45\%).

La conducta seguida fue de observación en $34 \operatorname{casos}(30,9 \%)$, que por mantenerse asintomá.

Tabla 1

Distribución según edad gestacional del total de racidos vivos vs. recién nacidos con poliglobulia*

\begin{tabular}{lcccc}
\hline & \multicolumn{2}{c}{$\begin{array}{c}\text { Total } \\
\text { nacidos vivos }\end{array}$} & \multicolumn{2}{c}{$\begin{array}{c}\text { Recién nacidos } \\
\text { poliglobulia }\end{array}$} \\
\cline { 2 - 6 } & NO & $\%$ & & \multicolumn{2}{c}{ No } & $\%$ \\
\hline RN Pret & 702 & $\lfloor 1,7$ & 28 & 25,5 \\
RNT & 5.250 & 87,6 & 80 & 72,7 \\
RN Post & 30 & 0,5 & 2 & 1,8 \\
TOTAL & 5.982 & 100,0 & 110 & 100,0 \\
\hline
\end{tabular}

* Hospital Clínico U.C. $1^{\circ}$ enero 1984 a 30 junio 1986.

Tabla 2

Distribución según peso para la edad gestional del total de nacidos vivos us. recién nacidos con poliglobulia*

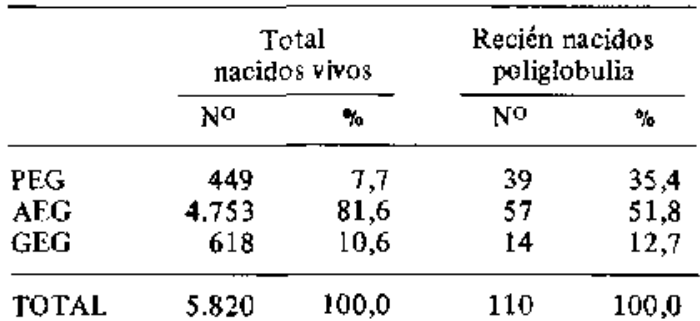

* Hospital Clínico U.C. $1^{\circ}$ enero 1984 a 30 junio 1986. 
Tabla 3

Sintomatología clínica 110 casos de poliglobulia neonatal

\begin{tabular}{lrrr}
\hline - ASINTOMATICOS: & $43(39,8 \%)$ & & \\
- SINTOMATICOS: & $67(60,2 \%)$ & & \\
\hline - SINTOMATOLOGIA & $\mathrm{N}^{*}$ & $\%$ \\
\hline & 51 & 78,4 \\
Rubicundez & 14 & 21,5 \\
Cianosis & 12 & 18,4 \\
Polipnea & 10 & 15,4 \\
Temblor & 8 & 12,3 \\
Hipotonía & 4 & 6,1 \\
Fenómenos vasomotores & & \\
(ptiapismo, alteraciones de To, etc.). & &
\end{tabular}

* Varios R.N. se presentaron con más de un síntoma.

Tabia 4

Hallazgos clínicos asociados a la poliglobulía neonatal

\begin{tabular}{lrr}
\hline PATOLOGIA ASOClADA & N & $\%$ \\
\hline - Hiperbilirrubinemia & 40 & 36,3 \\
- Hipoglicemia & 7 & 6,4 \\
- Infeción & 6 & 5,5 \\
- Trastornos de coagulación & 4 & 3,6 \\
- Hipocalcemia & 1 & 0,9 \\
- E.C.N. & 1 & 0,9 \\
- Rush alérgico & 1 & 0,9 \\
\hline
\end{tabular}

ticos se alimentaron sin presentar complicaciones. Se indicó fleboclisis más régimen cero durante 24 horas en $31(28,2 \%)$, todos éstos con una evolución favorable; y eritroféresis en 45 $(\mathbf{4 0}, 9 \%)$. Dentro de las eritroféresis realizadas, $11(24,4 \%)$ fueron por via umbilical y $34(75,6 \%)$ por via periférica. No hubo diferencias en cuanto a las complicaciones según la via de eritroféresis. Como hallazgos clínicos asociados se encontró hiperbilirrubinemia en 40 casos $(36,3 \%)$, hipoglicemia en $7(6,4 \%)$, infección en $6(5,5 \%)$ y otras que se muestran en la tabla 4.

Sólo en un caso se observó enterocolitis necrotizante (ECN), que corresponde al único caso fallecido $(0,9 \%)$.

Dieciséis $\mathrm{RN}(14,5 \%)$ no requirieron hospitalización; los 94 restantes permanecieron en promedio 4,56 dias; el grupo que requirió eritroféresis permaneció en promedio 7,6 dias en la unidad.

\section{DISCUSION}

La incidencia de poliglobulia varia según la altura sobre el nivel del mar donde se efectúa la determinación. En Denver, $1.612 \mathrm{~m}$ sobre el nivel del mar, se demostró una incidencia de $4 \%{ }^{10}$. A nivel del mar se han descrito incidencias de $1,8 \%$ a $2,2 \%^{11}$, similares a la obtenida en esta muestra.

Existe una clara relación entre poliglobulia y edad gestacional y en especial con la adecuación de peso para EG. La incidencia es mayor en nconatos PEG y GEG y es rarisima en recién nacidos de menos de 34 semanas de $\mathrm{EG}^{1,2,6}$. Nuestra serie muestra una incidencia cinco veces mayor en PEG en relación a recién nacidos AEG. Los GEG muestran una proporción mayor entre las poliglobulias que en la población global. Del nismo modo, la frecuencia de presentación de poliglobulia fue 25 veces mayor en los RN con sindrome de Down ${ }^{10,11}$.

El conocimiento de los grupos con riesgo de poliglobulia permite, mediante mediciones de hematocrito en las primeras horas de vida, detectar precozmente el problema ${ }^{3,12}$. En más de la mitad de los casos de esta serie el diagnóstico se hizo dentro de las dos primeras horas de vida.

En cuanto a la presentación clínica, la frecuencia de poliglobulia ssintomática fue similar a la de otras series ${ }^{6}$ como asimismo el tipo y frecuencia de los síntomas $1,3,5,10$.

Inicialmente empleamos la eritroféresis con plasma fresco de adultos, pero publicaciones recientes demuestran que la viscosidad de éste sería muy elevada para el $\mathrm{RN}^{3,7}$, por lo que dessde enero de 1987 la hacemos con solución $\mathrm{NaCl}$ al $9 \%$, sin registrar complicaciones a pesar de que con plasma no habíamos tenido efectos adversos. La vía periférica no ofrece mayores problemas y nos parece preferible, ya que es de más fácil acceso y requiere técnicas y materiales más simples que la umbilical.

Los hallazgos clinicos asociados no difieren en forma importante de otras series previamente publicadas ${ }^{1,3,6}$. La mortaljdad de la serje fue dada por un RN que tenía múltiples malformaciones que incidieron en su deceso y sufrió enterocolitis necrotizante.

El pronóstico a largo plazo depende de la causa de la poliglobulia, que puede condicionar factores que influyan en la evolución (asfixia, acidosis, etc.). Existen publicaciones que muestran mayor riesgo de problemas neurológicos y retraso de desarrollo en $\mathrm{RN}$ poliglobúlicos no tratados ${ }^{3,13}$; sin embargo, este punto permanece en discusión, ya que tambiến habría que considerar los riesgos inherentes al procedimiento. 
Esta revisión ratifica los factores de riesgo, manifestaciones clinicas y enfermedades asociadas a la poliglobulia y sugiere que la eritroféresis por vía periférica no difiere en eficacia de la vía umbilical, es más sencilla de realizar y tiene menos complicaciones potenciales.

\section{RESUMEN}

Se revisaron retrospectivamente 110 casos de poliglobulia neonatal entre enero de 1984 y junio de 1986: $35,4 \%$ eran pequeños para edad gesta. cional y $12,7 \%$ grandes para edad gestacional. En las afecciones maternas destacan el síndrome hipertensivo del embarazo $(17,2 \%)$ y la diabetes gestacional $(6 \%)$. En el recién nacido el síndrome de Down se presentó en $6 \operatorname{casos}(5,2 \%)$. En $52,8 \%$ de los casos el diagnóstico fue precoz $(0 \mathrm{a} 2 \mathrm{~h} \mathrm{de}$ vida), 60,2\% tuvieron sín tomas, destacando rubjcundez $(78,4 \%)$ y con menor frecuencia cianosis, temblor, polipnea e hipotonía: 45 casos $(40,9 \%)$ requirieron eritroféresis, de los cuales $11(24,4 \%)$ se hicieron por vía umbilical y $34(75,6 \%)$ por vía periférica. Las enfermedades asociadas más frecuentes fueron hiperbilirrubinemia en 40 casos $(36,3 \%)$, hipoglicemia en $7(6,4 \%)$ y sepsis en 6 $(5,5 \%)$. El único paciente fallecido de la serie correspondió a un niño con múltiple patología agregada, anomalías congénitas y ECN.

\section{REFERENCLAS}

1. Oski Naiman: Problemas hematológicos en el Recién Nacido. 3a. Ed. Buenos Aires, Ed. Médica Panamericana S.A. 1984; 101-110.
2. Black $Y, D$., Lubchenco $L_{\text {..: }}$ Neonatal polycythemia and hyperviscosity. Pediatr Clin North Am 1982; 29: $1137-1148$.

3. Oh $W$ : Neonatal polycythemia and hyperviscosity. Pediatr Clin North Am 1986; 33 : 523-532.

4. Gross G.P., Hathaway W.E., McGaughey H.R.: Hyperviscosity in the neonate. J Pediatr 1973:82 1004-1012.

5. Ramamurthy R.S., Brans $Y . W$.: Neonatal polycy themia: I criteria for diagnosis and treatment. Pediatrics 1981 ; 68 (2): 168-174.

6. Wiswell T.E., Cornish M.J., Northam R.S.: Neonatal polycythemia: frequency of clinical manifestations and other associated findings. Pediatrics 1986; 78 (1): 26-30.

7. Linderkamp $O$., Veersmold K.P., Retke $K$.: Contributions of red cells and plasma to blood viscosity in preterm and full-term infants and adults. Pediatries $1984 ; 74: 45-51$.

8. Brans Y.W. Shannon O.L. Rantamurthy R.S. Neonatal polycythomia: II plasina blood, and red cell volume estimates in relation to hematocrit levels and quality of intrauterine growth. Pcdiatrics. 1981; 68: 175-182.

9. Juez G. Ventura-Juncd P. Lucero E.F.: "Crecimiento intrauterino en un grupo seleccionado de recién naciáos chilenos. "Subdiagnóstico de retardo de crecimiento intrauterino en Chile?". Rev Med Chile 1984; 112: 759-764.

10. Wirth F.H., Goldberg K.E., Lubchenco L.: "Neonatal hyperviscosity: I Incidence". Pediatrics 1979, 63: 833-836.

11. Stevens $K$., Wirth F.H.: Incidence of neonatal hypervisoosity at sea level. J Pediat $1980 ; 97$ : 118-119.

12. Shohat $M$., Merloab $P$., Keisnep $S$. "Neonatal polycythemia: I Farly diagnosis and incidence relating to time of sampling". Pediatrics 1984; 73: $7-10$.

13. Block V.O., Lubchenco L.O., Luckey D.W. et at: Developmental and Neurologie sequelac of Neonatal hyperviscosity syndrome. Pediatrics 1982; $69: 426-431$. 